Krämer, Sybille/Schmidt, Sibylle/Schülein, Johannes-Georg: Philosophie der Zeugenschaft. Eine Anthologie. Münster: Mentis 2017. 243 Seiten. [978-3-95743-078-6]

Rezensiert von Suzana Alpsancar (BTU Cottbus Senftenberg)

Sybille Krämer, Sibylle Schmidt und Johannes-Georg Schülein versammeln in ihrer Anthologie Philosophie der Zeugenschaft zentrale Quellen aus Philosophiegeschichte und zeitgenössischen Debatten zum Phänomen der Zeugenschaft. Drei Textgruppen werden präsentiert: zunächst Autoren der Aufklärung (Arnauld, Locke, Hume, Reid, Kant, Bentham), dann Auszüge der jüngeren, vor allem im angloamerikanischem Raum geführten testimony-Debatte (C. A. J. Coady, Elizabeth Fricker, Jennifer Lackey, Miranda Fricker und Richard Moran), die hier erstmalig ins Deutsche übersetzt sind (ebenso wie die Auszüge von Reid). Dieser Gruppe ist ein kurzer Auszug aus Wittgensteins Über Gewissheit vorangestellt, der für sich selbst steht, da die testimony-Debatte ihrem Selbstverständnis nach erst mit C. A. J. Coadys Monographie Testimony. A philosophical study von 1992 ihren Anfang nahm. Es folgt eine dritte, vom Umfang her kleinere Gruppe, die eine kontinentale Debatte um Zeugenschaft bekunden soll (Jacques Derrida, Giorgio Agamben, Paul Ricoeur und Avishai Agalit). Diese Quellen sind von den Herausgeber*innen, die mehrfach zum Thema publiziert haben (z.B. Schmidt, Krämer und Voges 2010), freilich nicht bloß editiert, sondern in ein bestimmtes Licht gerückt, in dem sich ihr eigenes Programm einer Philosophie der Zeugenschaft spiegelt. Die Leser*innen finden in der Einleitung sowie in den kurzen Kommentaren zu den Quelltexten daher auch einen Beitrag zur aktuellen Debatte um Zeugenschaft. Ihre zentrale These lautet, die testimony-Debatte verkürze das Problem der Zeugenschaft und müsse philosophiehistorisch korrigiert sowie sozialphilosophisch-ethisch erweitert werden. Der Zuschnitt der vorliegenden Textsammlung lädt so dazu ein, die Philosophie der Zeugenschaft, so wie sie die Herausgeber*innen entwickeln, anhand ihrer Quellen nachzuvollziehen. Ich folge dieser Spur.

\title{
Die testimony-Debatte
}

Der erste Schritt hierzu ist folgende Charakterisierung des „Narrativs“ (13) der testimony-Debatte: Im Fokus stehe erstens die Frage nach dem epistemischen Status testimonialen Wissens. Diesen rein erkenntnistheoretischen Zugriff auf Zeugenschaft wollen die Herausgeber*innen um einen sozialphilosophisch-ethischen erweitern (siehe auch Schmidt 2015). Zweitens denke die 
Debatte Zeugenschaft weitestgehend im Paradigma der ,alltäglichen Informationsübermittlung“ (13), womit drittens eine auf die Philosophie der Aufklärung limitierte Rezeption der Geschichte der philosophischen Zeugnisreflexion einhergehe, die zudem den Beitrag der Aufklärung zum Thema verzerre, wenn sie diese auf das Denkmuster eines „epistemischen Individualismus" (9) verenge.

In der testimony-Debatte schreibt man das Denkmuster eines epistemischen Individualismus für gewöhnlich der gesamten Aufklärungsphilosophie zu und scheut sich kaum, diese Zuschreibung zu einer Charakterisierung der gesamten abendländischen Philosophie (von Platon bis Wittgenstein) zu generalisieren (diesem Narrativ folgen auch Krämer, Schmidt und Schülein erst einmal: 7 und 10). Dabei scheint man sich mit einer Karikatur dieser Position zufrieden zu geben, wie man sie bei E. Fricker findet:

Dieser Idealtypus verlässt sich, wo es um Wissen geht, einzig und allein auf sich selbst. Er stützt sich nicht auf die Aussagen anderer, sondern akzeptiert nur das, was er selbständig herausgefunden hat, und er vertraut dabei allein seinen kognitiven Fähigkeiten, seinem Vermögen, etwas zu untersuchen und Schlüsse zu ziehen. Eben dieses Ideal und diese Methode hat Descartes ausdrücklich in seinen Mediationen (1641) vertreten. (Fricker, zitiert auf 112)

Da hiernach Wissen aus zweiter Hand nur dann gerechtfertigt sei, wenn es auf „die individuellen Ressourcen von Wahrnehmung, Gedächtnis und induktivem Schlussfolgern“ (14) zurückführbar sei, bezeichnet man die Position des epistemischen Individualismus auch als Reduktionismus. Coady meint, eine solche „Reduktionische These“ (98) in Humes Bemerkungen über Wunder in Untersuchung über den menschlichen Verstand (1748) sowie in Traktat über die menschliche Natur (1739/40) vorzufinden. Nach Coady gilt Humes Position gar als ein globaler Reduktionismus, weil dieser testimoniales Wissen unter allen Umständen auf die genannten primären Wissensquellen zurückführen wolle. Dagegen macht Coady unsere unhintergehbare Abhängigkeit von Aussagen anderer in „unserem alltäglichen Handeln“ (96) stark. Außerdem hält er einen solchen Reduktionismus für logisch zirkulär und praktisch undurchführbar: Die Aussage der anderen zu überprüfen setze schließlich ein Wissen über den bezeugten Sachverhalt voraus, über welches man aber erst durch die Zeugenaussage verfügt. Und selbst wenn sich der testimonial erhobene Wissensanspruch prinzipiell durch eine eigene Beobachtung oder ein Schlussfolgern verifizieren ließe, würden wir uns handlungsunfähig machen, versuchten wir, eine solche Prüfung auch nur annähernd durchzuführen. Coady plädiert deswegen dafür, „dass die Evidenz von Zeugnissen eine eigene, grundlegende 
Kategorie von Evidenz bildet, die sich nicht auf andere fundamentale Kategorien wie Beobachtung oder deduktive Schlussfolgerung zurückführen lassen“ (106). Philosophiehistorisches Vorbild für Coadys Anti-Reduktionismus ist Thomas Reid (57-64). Von diesem Gegenpaar ausgehend versuche die Debatte zu klären, wie ein angemessenes Verhältnis zwischen der „Anerkennung der Sozialität unserer Erkenntnis“ (111) und dem - von der Aufklärung ja durchaus auch kritisch und emanzipativ gedachtem - Credo des „Selberdenken" (11) auszusehen habe. Lackey macht darauf aufmerksam, dass man für die Frage nach Testimonialwissen sowohl Sprecher als auch Hörer berücksichtigen muss (129ff.), M. Fricker betont die „politische Dimension der sozialen Epistemologie“ (149), Moran „beschreibt das Bezeugen als einen spezifischen Sprechakt", bei dem man auch die „interpersonale[n] Dimension“ der „Praxis des Bezeugens“ (165) berücksichtigen müsse.

Die Herausgeber*innen verdichten diese Diskussion der testimony-Debatte systematisch zur Leitdifferenz von „Mündigkeit versus Unmündigkeit“ (9) und plädieren für eine vermittelnde Position, die sowohl unserer epistemischen Abhängigkeit von Zeugenschaft als auch dem emanzipativen Anspruch der Aufklärung gerecht werde (19-20). Der zweite Schritt hierzu ist ihre historische Korrektur der Rezeption der Aufklärungsphilosophie in der testimonyDebatte.

\section{Philosophiehistorische Korrektur der testimony-Debatte}

Um das testimony-Narrativ philosophiehistorisch zu korrigieren, legen die Herausgeber*innen die Textstellen vor, die in der Debatte referiert werden, und ergänzen diese um weitere Auszüge derselben Autoren, die deren breitere Auseinandersetzung mit dem Phänomen der Zeugenschaft dartun. Ein Beispiel dafür stammt aus Humes Schrift Über das Studium der Geschichte (1741):

Und tatsächlich, wenn wir die Kürze des menschlichen Lebens betrachten und unser begrenztes Wissen sogar um das, was in unserer eigenen Zeit geschieht, müßten wir einsehen, daß unser Verstehen immer kindlich bliebe, gäbe es nicht diese Erfindung, die unsere Erfahrung auf alle vergangenen Zeiten und auf die entferntesten Nationen hin erweitert; sodaß diese so viel zum Fortschritt unseres Wissens beitragen, als würden sie uns tatsächlich vor Augen liegen. (54)

Das Überführen des historisch eindimensionalen Zugriffs gelingt mit Bravour. In Lockes Versuch über den menschlichen Verstand (1690) lesen wir, dass in vielen Situationen die Gewissheit einer Erkenntnis nicht unmittelbar evident ist und dass man Behauptungen anderer erst nach „angemessener Abwägung aller Umstände [...] verwerfen oder annehmen“ (38) sollte. Hierzu sei man auf 
kontextuelles und situatives Hintergrundwissen angewiesen, wie Locke mit der Anekdote des Königs von Siam plausibilisiert:

So ging es einem holländischen Gesandten, der den König von Siam von den Eigentümlichkeiten Hollands unterrichtete, nach denen dieser sich erkundigt hatte. Er erzählte ihm unter anderem, daß das Wasser in seiner Heimat bei kaltem Wetter zuweilen so fest werde, daß die Menschen darauf umhergehen könnten, ja, daß es einen Elefanten tragen würde, wenn einer vorhanden wäre. Darauf erwiderte der König: „Bis jetzt habe ich die seltsamsten Dinge geglaubt, die sie mir erzählt haben, weil ich Sie für einen besonnene und ehrlichen Mann hielt, jetzt aber bin ich gewiß, daß Sie lügen.“ (38-39)

Offenkundig passen solche Erwägungen Lockes nicht zu der Einschätzung desselben, die man z.B. bei E. Fricker nachlesen kann:

[...] John Locke hat der Auffassung widersprochen, , die durch unseren Kopf schwirrende Meinung anderer' stellten Wissen dar (1690). Der ganz und gar autonome Wissende wird keine Aussage billigen, sofern er nicht über die sie begründende Evidenz verfügt. Er wird also nichts auf der Grundlage des Wortes anderer akzeptieren, selbst wenn er Belege dafür hat, dass sie hinsichtlich des fraglichen Gegenstands vertrauenswürdig sind. (112)

Wie wir sehen, hat E. Fricker ihr Urteil nicht aus dem ,ganzen' Locke gezogen, sondern aus einigen seiner (Zwischen-) Überlegungen. Doch was folgern wir daraus? Müsste man in philosophiehistorischer Redlichkeit nicht nach den Gründen für diese scheinbare Unstimmigkeit in Lockes Werk suchen? Sollte man Leser*innen nicht dazu anregen, Lockes Überlegungen zur Zeugenschaft aus der begrifflichen Konstellation seines eigenen Werks heraus nachzuvollziehen? Ursula Renz erinnert zum Beispiel daran, dass der argumentative Gegner diverser aufklärerischer Denker historisch nicht eine Sozialphilosophie der Praxis war, ihre Gegner waren stattdessen (Renz verweist auf Platon, Descartes und Hume) „die Sophisten, der scholastische Autoritätsbeweis sowie der auf religiöser Überlieferung basierende Wunderglaube“ (Renz 2009: 248). Renz macht auf die begriffliche Unbestimmtheit der Rede vom epistemischen Individualismus aufmerksam (siehe auch Schmidt und Scholz 2010), die in ähnlicher Schärfe Alex Gelfert (2010: 64) attackiert, der im Zerrbild des epistemischen Individualismus einen Strohmann erkennt, gegen den man die eigenen Position einer social epistemology in Szene setze. Es ist bedauerlich, dass die Herausgeber*innen auf derartige Kritik zwar verweisen, diese jedoch nicht in ihre eigene Auseinandersetzung einbeziehen. Ihre „historische Korrektur“ scheint sich mit dem Nachweis zufrieden zu geben, dass die Aufklärungsphilosophie „das Phänomen der Zeugenschaft und des Zeugniswissens substan- 
ziell und vielseitig reflektiert" (9) hat und man bei den Aufklärungsphilosophen viele „neue Impulse“ (9) für die heutige Diskussion finden kann. Mir scheint es verkürzt, der testiminoy-Debatte philosophiehistorisch nur vorzuwerfen, sie haben die falschen oder schlicht zu wenige Textstellen zu Rate gezogen. Ist nicht das Modell eines epistemischen Individualismus, wie es E. Fricker präsentiert (und das wohl auf J. L. Mackies The Possibility of Innate Knowledge zurückgeht: vgl. Coady, 96), viel grundsätzlicher zu hinterfragen? Was kann überhaupt sinnvollerweise mit dem Attribut des Individualismus in Bezug auf epistemische Fragen gemeint sein?

Obwohl die Herausgeber*innen diese Frage nicht stellen, finden sich in ihrer Rekonstruktion der Debatten einige Antwortoptionen. Gehen wir von dem reduktionistischen Ansatz aus, Wissensansprüche könnten nur dann verifiziert werden, wenn man sie (a) auf eigene Beobachtung zurückführen kann oder wenn man sich die Behauptung (b) eigens durch Schlussfolgern erschlieBen kann. Was könnte an diese beiden Prüfverfahren ,individualistisch' genannt werden? Da die „theoretische Notwendigkeit“ (Derrida: 199), mit der ein Schluss gilt/nicht gilt, uns von der Person, die den Schluss vollzieht, absehen lässt, scheint es unpassend, Schlussverfahren (wie auch anschauliche Beweise oder Folgen einer Argumentation) als individualistisch zu bezeichnen. Wie Derrida betont (199), brauchen uns Wissenschaftler*innen nicht darum bitten, ihnen zu glauben, wenn sie uns ihre Schlüsse vorführen, weil wir davon ausgehen, dass diese auf methodischem Wege erbracht und somit intersubjektiv nachvollziehbar sind. Dennoch bleibt Evidenz auch personengebunden. Ein fruchtbarer Zweig dieser Debatte könnte folglich dahingehend wachsen, die jeweiligen Verbindungen, in denen diese Gebundenheit mit dem Transpersonalen von Evidenz und Wissen jeweils steht, genauer zu erkunden.

Bleibt das Prüfverfahren (a) der eigenen Beobachtung. Hier könnte die Zuschreibung, individualistisch'so gemeint sein, dass die eigenen Beobachtung immer eine je individuelle sei, weil sie jedes Individuum für sich macht. Es stimmt zwar, dass wir unsere Beobachtungen und Erfahrungen je selbst machen, dennoch folgt hieraus nicht, dass diese privat oder singulär wären. Hier müsste präzisiert werden, was mit ,individualistisch' genau gemeint sein soll. Auch wäre in Bezug auf die Rezeption der Aufklärungsphilosophie zu bedenken, dass wohl die meisten Philosophen (von Platon bis Wittgenstein) Erfahrung und Beobachtungsaussagen für begrifflich vermittelt halten und diese schon allein deswegen ebenso wenig wie Sprache privat sein können. Bei Hume und Kant steht der Erfahrungsbegriff zudem terminologisch für die Gleichförmigkeit natürlicher Phänomene. Die Regelmäßigkeit (bei Kant: 
der Gesetzescharakter) von Phänomenverbindungen und Ereignisfolgen ist doch dasjenige, was verbirgt, dass eine Erforschung der Natur/Welt der Erscheinung überhaupt als möglich gedacht werden kann. Wenn Beobachtbares/Erfahrung aber prinzipiell intersubjektiv nachvollziehbar ist, bleibt unklar, was die Zuschreibung individualistisch hier fassen soll.

Die Kennzeichnung bezieht sich letztlich wohl am ehesten auf eine Einstellung, für die die Herausgeber*innen die griffige Formel vorschlagen: „Do it yourself‘ (10). Nicht die Verifikations-/Falsifikationsverfahren selbst wären individualistisch, sondern die Personen/Forscher*innen, die nur glauben, was sie selbst geprüft haben. Dass auch das Selbermachen sozial vermittelt bleibt, haben wir oben angedeutet. Individualistisch wären die Skeptiker*innen und Eigenbrötler*innen, die sich zurückziehen und niemanden vertrauen wollen. Um im so verstandenem Individualismus ein Gegenprinzip zu einer Praxis des Miteinanders zu finden, könnte man die Formel ergänzen: Do it always yourself withouth any help! Dass dieser Imperativ wenig praxistauglich ist, versteht sich von selbst.

Für weiterführende Diskussionen scheint es sinnvoll, die Figur des ,epistemischen Individualismus' grundsätzlicher zu problematisieren: Bezieht sich die Zuschreibung auf Genese oder Geltung von Wissen oder den Umgang mit Wissensansprüchen? Was genau soll hier als, individualistisch' gekennzeichnet werden? Heißt dabei individualistisch immer soviel wie ,nichtsozial vermittelt'? In welchem Sinn kann dies überhaupt eine sinnvolle $\mathrm{Zu}-$ schreibung sein? Auf welchem Wissensbegriff fußt die Diskussion (wohl einem propositionalem) und inwiefern strukturiert diese Vorstellung von Wissen die Auseinandersetzung?

Die philosophiehistorische Korrektur der Herausgeber*innen zeigt letztlich mehr, als dass sich bei den Aufklärungsphilosophen auch sozialepistemologische Motive finden und die testimony-Debatte diese missverständlich rezipiert. Man fühlt sich hier indirekt aufgefordert, nach den wissenshistorischen Konstellationen $\mathrm{zu}$ fragen, in denen verschiedenen Kriterien von Evidenz eine je andere Bedeutsamkeit zukommen kann und die Spannung zwischen Genese und Geltung von Wissen jeweils anders fließen mag. Diese Perspektive einer stärker historischen Lesart wird im abschließend Plädoyer dadurch konterkariert, dass die Herausgeber*innen Einseitigkeiten der Debatten und damit ihre Frontlinien (testimony-Debatte vs. Aufklärung, testimony-Debatte vs. kontinentale Ethik) einen Tick zu stark betonen. Gleichzeitig leisten ihre kompakten „Narrative“ der Diskussionszusammenhänge unbestritten einen gelungen Einstieg in die Philosophie der Zeugenschaft. 


\section{Mündigkeit versus Unmündigkeit?}

In ihrem dritten Schritt plädieren die Herausgeber*innen dafür, in einer Philosophie der Zeugenschaft sowohl den emanzipativen Anspruch der Aufklärung als auch die Einsicht in unsere epistemische Abhängigkeit der social epistemology zusammen zu bringen. Wenn sie dies als Widerstreit zwischen „Mündigkeit und Unmündigkeit" (9) deklarieren, ja die These formulieren, dass beide Seiten in Form eines Dilemmas konstitutiv zu einer Philosophie der Zeugenschaft gehörten (9), schreiben die Herausgeber*innen das Zerrbild der Aufklärungsphilosophie in gewisser Weise fort. Denn so werden auf der einen Seite Mündigkeit, Autonomie, Unabhängigkeit und Individualismus und auf der anderen Seite Unmündigkeit, Heteronomie, Abhängigkeit und Sozialität der Praxis in eins gesetzt und beide Seiten in Opposition zueinander gebracht. Das ergibt ein undifferenziertes Bild, in dem zum Beispiel Kants Autonomiebegriff mit Unabhängigkeit gleichzusetzen wäre. Ist es aber nicht verkürzt, Kants „Wahlspruch der Aufklärung“ saper aude! (9) kontextlos in die Formel do-it-yourself! (10) zu übersetzen? Die weitere Forschung, welche die Herausgeber*innen anregen wollen, könnte sich daran setzen, das Problemfeld begrifflich präziser zu beschreiben und im Lichte der reklamierten „Uneindeutigkeit der Figur des Bezeugens" (9) zu sortieren. Eine Spur, mit der die historische Korrektur fortgesetzt werden könnte, haben die Herausgeber*innen eingangs gelegt, wenn sie darauf hinweisen, dass in der neuzeitlichen Philosophie der Aufklärung das „Verhältnis von epistemischer Abhängigkeit und Autonomie“ (8) bereits thematisiert wurde. Inwiefern gleicht und unterscheidet sich also diese Fragestellung der Aufklärung von der heutigen, durch die testimony-Debatte konstruierten?

Ein entscheidender Unterschied zwischen Aufklärung und testimony-Debatte scheint mir darin zu liegen, dass erstere sich primär für die Erfahrbarkeit und Erforschbarkeit der Natur seitens der Gelehrtenwelt (also für Wissenschaft) und letztere primär für eine rationale Rekonstruktion des Austauschs von Wissen im Alltag interessiert. Da die Wissenschaften einen recht eigenwilligen Begriff und Zugang zum Wissen verbürgen, sollte man die Vergleiche zwischen „Lebenspraxis“ und „wissenschaftlicher Arbeit“ (10) vielleicht nicht so bruchlos heranziehen, wie es die Herausgeber*innen teilweise tun. Fragen nach dem Status von Zeugniswissen stellen sich in beiden Bereichen auf verschiedene Weise, da die Wissenschaften eine kritische Grundhaltung aus Erkenntnisinteresse anders kultivieren als es für andere Lebensbereiche klug und praktikabel wäre. Wie genau sich das Ineinander dieser kritischen Grundhaltung und der Praxis des miteinander Arbeiten und Lernens etc. vollzieht, ließe 
sich vielleicht im Austausch mit der Wissenschaftsforschung näher erforschen, die ihrerseits angesichts der Historisierung und Soziologisierung unseres Wissenschaftsverständnis seit einigen Jahrzehnten über das Verhältnis von Genese und Geltung von Wissensansprüchen sowie von Theorie (theoretischer Notwendigkeit) und Praxis diskutiert. Angeleitet durch eine Phänomenologie der verschiedenen Zeugnis-Konstellationen, wie sie zum Beispiel Krämer (2008) ausarbeitet, könnte man (auch in Bezug auf verschiedene Wissenstypen wie Wahrnehmen, Erinnern, Können, Kenntnisse, Begründen) weiter fragen, inwiefern hier die Prinzipen von Mündigkeit/Selberdenken und die Realität der gegenseitigen Abhängigkeit tatsächlich ein Dilemma bilden und wo sie produktiv ineinander spielen.

\section{Episteme und Ethik}

Im vierten Schritt werben die Herausgeber*innen dafür, eine Philosophie der Zeugenschaft epistemisch und ethisch anzugehen. Den Weg dazu sehen sie durch die kontinentalen Beiträge bereitet. Während Ethik derzeit häufig als Reflexion und Rechtfertigung moralischer Prinzipien, Maximen und Normen in den Kategorien von gut und schlecht verstanden wird, verstehen sie Ethik im Sinne einer Sozialphilosophie der Anerkennung. An dieser Stelle hätte man auf Beiträge angelsächsischer Autoren zur Ethik der Zeugenschaft hinweisen können (z.B. Richard Moran). Auch hätte man hier die Kernbegriffe, von denen diese Diskussionen (möglicherweise) strukturiert sind, grundsätzlich problematisieren können; etwa den Begriff moralischen Wissens beziehungsweise das Verhältnis von sogenannten praktischen und theoretischen Wissensansprüchen (siehe zum Beispiel Heusinger von Waldegge 2017).

Die kontinentale Debatte erläutern die Herausgeber*innen zur Verdeutlichung ebenfalls über ein Narrativ: Ihr Paradigma sei das „Opferzeugnis“ (17) (Opferzeug*in) und damit die „Person“ an Stelle der „Aussage“ (17). Wo die testimony-Debatte um die Rechtfertigung des mitgeteilten Sachverhaltes kreise, gehe es hier um Interpersonalität, Vertrauen, Integrität und Anerkennung von Personen, also um eine ethische wie politische Dimension, welche die Praxis des Bezeugens in die Nähe der Praxis des Versprechens rücke. Die Zeugenreden stünden zweitens nicht in einem „Regime der Wahrheit als propositionaler, an Evidenz und Rechtfertigung geknüpfter Rede“ (17), sondern in einem Regime der Anerkennung: Anerkennung des erfahrenen Leids und damit der Opfer als Personen. Zudem stehe das Nachvollziehbarmachen dieser subjektiven Erlebnisse, an denen immer etwas Unnachvollziehbares haften bleibt, im Zentrum. In dieser Hinsicht kann Agamben den Aus- 
schwitz-Überlebenden Primo Levi als „ein perfektes Beispiel des Zeugen“ (207) sehen. Mit der Person rücke außerdem das Körperliche der BezeugensPraxis in den Blick, der so über das Aussageförmige des Paradigmas der Mitteilung hinausgehe; auch das Schweigen kann Teil des Bezeugens sein (Derrida: 199).

Die Praktiken des alltäglichen Bezeugens und der Opferzeugen sollen den Herausgeber*innen zufolge nicht bloß addiert werden. Vielmehr soll die Zusammenstellung der angelsächsischen mit der kontinentalen Debatte die zweite philosophische These belegen helfen, dass der Zeugenschaft nicht nur das Dilemma von Mündigkeit und Unmündigkeit eingeschrieben sei, sondern eben auch das Spannungsverhältnis von „Episteme versus Ethik“ (9). Dass die Herausgeber*innen diese beiden Narrative zusammenbringen, erscheint vielversprechend. Doch gerne hätte man noch mehr darüber erfahren, wie sich die so provozierte gegenseitige „Befruchtung“ (15) vorstellen (z.B. hätte man einen Hinweis setzen können, dass Derrida, Ricoeur wie Lackey und Moran das Dialogische der Zeugenschaft betonen). Der Weg über die Sprechakttheorie, den sie hier andeuten, bleibt vage, fast floskelhaft, wenn es abschließend heißt:

Einen Grund unserer Erkenntnis bilden neben individuellen Wahrnehmungsurteilen und Schlussfolgerungen eben auch soziale (Vertrauens-)Beziehungen, die ethisch und politisch reflektiert werden können. (19)

Warum halten sich die Herausgeber*innen hier zurück? Krämer (2008: 223260) hat ja bereits am Paradigma des Gerichts-Zeugens eine Figur durchdacht, die grundlegend eine Instanz des Versprechens und Vertrauens unter Eid bildet und hierin einem Wahrheits- (oder zumindest einem Wahrhaftigkeits-)anspruch gerecht werden muss. Meint die These, dass Episteme und Ethik beim Phänomen der Zeugenschaft immer so zusammen kommen, wie es das Paradigma des Gerichtszeugen zeigt? Soll diese (vielleicht mit Ricoeur: 222 und gegen Agalit: 237) für das Phänomen der Zeugenschaft insgesamt gelten?

Wie die Anthologie eindrücklich verdeutlicht, geht eine Philosopbie der Zeugenschaft nicht in der Frage nach dem epistemischen Status testimonialen Wissens auf. Ob man der Heterogenität des Phänomens aber dadurch gerecht werden kann, dass man der Logik einer Gegenüberstellung von Mündigkeit und Unmündigkeit sowie Episteme und Ethik verhaftet bleibt, scheint mir ungewiss. Die testimony-Debatte bleibt so jedenfalls das Gravitationszentrum dieser Philosophie der Zeugenschaft. Es ist das Verdienst der Herausge- 
ber*innen, zentrale Quellen und Debattenbeiträge der Philosophie der Zeugenschaft in einer Hand zugänglich gemacht zu haben und in den aktuellen Diskussionstand einzuführen. Da sie die Debatten zudem nicht bloß editorisch, sondern auch programmatisch kommentieren, fühlt man sich nicht nur durch ihre Quellen, sondern auch durch ihre Thesen zum Weiterdenken animiert. Indem man dieser Einladung folgt, zeigt sich wohl, was die Herausgeber*innen uns mit auf dem Weg geben wollten: dass zu einer Einschätzung der Praxis des Bezeugens immer beides gehören muss, vertrauensbasierter Austausch und kritisches Selberdenken.

\section{Literatur}

Gelfert, Axel. „Hume on Testimony Revisited.“ Logical Analysis and History of Philosophy 13 (2010), 60-75.

Heusinger von Waldegge, Florian. Zum Problem moralischen Wissens. Ethischer Relationalismus in Anschluss an Hegel. Bielefeld: transcript 2016.

Krämer, Sybille. „Medium, Bote, Übertragung. Kleine Metaphysik der Medialität." Frankfurt am Main: Suhrkamp, 2008.

Schmidt, Frederick F. und Oliver R. Scholz. „Introduction: The History of Social Epistemology.“ Episteme 7.1 (2010), 1-6.

Schmidt, Sybille, Sybille Krämer, und Ramon Voges (Hg.). Politik der Zengenschaft. Zur Kritik einer Wissenspraxis. Bielefeld: transcript, 2010.

Schmidt, Sybille. Ethik und Episteme der Zeugenschaft. Konstanz: Konstanz University Press, 2015.

Scholz, Oliver R. „Von Rom, den Antipoden und von Wundern - Das Zeugnis anderer in Logiken der Neuzeit." In Unsicheres Wissen. Skeptizismus und Wahrscheinlichkeit 1550-1850, hg. von Carlos Spoerhase, 245-267. Berlin: Walter de Gruyter, 2009.

Renz, Ursula. „Warum selber denken? Zum Problem und Begriff des epistemischen Individualismus.“ Analyse und Kritik 31.2 (2009), 243-259. 